\title{
NORMAL FORMS ON CONTRACTING FOLIATIONS: SMOOTHNESS AND HOMOGENEOUS STRUCTURE
}

\author{
BORIS KALININ AND VICTORIA SADOVSKAYA*
}

\begin{abstract}
In this paper we consider a diffeomorphism $f$ of a compact manifold $\mathcal{M}$ which contracts an invariant foliation $W$ with smooth leaves. If the differential of $f$ on $T W$ has narrow band spectrum, there exist coordinates $\mathcal{H}_{x}: W_{x} \rightarrow T_{x} W$ in which $\left.f\right|_{W}$ has polynomial form. We present a modified approach that allows us to construct maps $\mathcal{H}_{x}$ that depend smoothly on $x$ along the leaves of $W$. Moreover, we show that on each leaf they give a coherent atlas with transition maps in a finite dimensional Lie group. Our results apply, in particular, to $C^{1}$-small perturbations of algebraic systems.
\end{abstract}

\section{INTRODUCTION}

The theory of normal forms of maps plays an important role in dynamics and goes back to the works of Poincare and Sternberg [St57]. Normal forms at fixed points and invariant manifolds have been extensively studied [BKo]. More recently, the theory of non-stationary linearizations and, more generally, normal forms was developed in the context of diffeomorphisms with contracting foliation [KtL91, GKt98, G02, Fe04, S05]. It proved useful in the study of smooth dynamics and rigidity for dynamical systems and group actions exhibiting various forms of hyperbolicity, see for example KtSp97, KS03, KS06, F07, FFH10, GoKS11, KKtR11, FiKSp11.

Let $f$ be a $C^{\infty}$ diffeomorphism of a compact smooth manifold $\mathcal{M}$, and let $W$ be a continuous invariant foliation with $C^{\infty}$ leaves which is contracted by $f$, that is $\left\|\left.D f\right|_{T W}\right\|<1$ in some Riemannian metric. The goal of the normal form theory in this setting is to find a family of diffeomorphisms $\mathcal{H}_{x}: W_{x} \rightarrow T_{x} W$ such that the maps

$$
\tilde{f}_{x}=\mathcal{H}_{f x} \circ f \circ \mathcal{H}_{x}^{-1}: T_{x} W \rightarrow T_{f x} W
$$

are as simple as possible, for example linear maps or polynomial maps in a finite dimensional Lie group. The maps $\mathcal{H}_{x}$ should depend well on $x$ and ideally form a good atlas on each leaf of $W$. For technical reasons, it is often easier to operate with marked leaves, which can be identified with the tangent spaces $T_{x} W$, producing an extension of the original system to $T W$. The results in [KtL91, GKt98, G02] are stated in such a setting, but most applications come from the foliated systems.

\footnotetext{
* Supported in part by NSF grant DMS-1301693.
} 
Non-stationary linearization, i.e. existence of $\mathcal{H}_{x}$ so that $\tilde{f}_{x}$ in (1.1) are linear, was first established by Katok and Lewis for one-dimensional $W$ KtL91]. For higherdimensional foliations under a uniform 1/2 pinching assumption, non-stationary linearization follows from results of Guysinsky and Katok GKt98 or from results of Feres in [Fe95, where a differential geometric point of view was developed. Under a weaker assumption of pointwise $1 / 2$ pinching, it was obtained in [S05] and additional properties were established in [KS06]. These results are summarized below.

Non-stationary linearization. Suppose that $\left\|\left.D f\right|_{T W}\right\|<1$, and there exist $C>0$ and $\gamma<1$ such that for all $x \in \mathcal{M}$ and $n \in \mathbb{N}$,

$$
\left\|\left(\left.D f^{n}\right|_{T_{x} W}\right)^{-1}\right\| \cdot\left\|\left.D f^{n}\right|_{T_{x} W}\right\|^{2} \leq C \gamma^{n} .
$$

Then for every $x \in \mathcal{M}$ there exists a $C^{\infty}$ diffeomorphism $\mathcal{H}_{x}: W_{x} \rightarrow T_{x} W$ such that

(i) $\mathcal{H}_{f x} \circ f \circ \mathcal{H}_{x}^{-1}=\left.D f\right|_{T_{x} W}$,

(ii) $\mathcal{H}_{x}(x)=0$ and $D_{x} \mathcal{H}_{x}$ is the identity map,

(iii) $\mathcal{H}_{x}$ depends continuously on $x \in \mathcal{M}$ in $C^{\infty}$ topology,

(iv) Such a family $\mathcal{H}_{x}$ is unique and depends smoothly on $x$ along the leaves of $W$,

(v) The map $\mathcal{H}_{y} \circ \mathcal{H}_{x}^{-1}: T_{x} W \rightarrow T_{y} W$ is affine for any $x \in \mathcal{M}$ and $y \in W_{x}$. Hence the non-stationary linearization $\mathcal{H}$ defines affine structures on the leaves of $W$.

For higher-dimensional $W$ without $1 / 2$ pinching there may be no smooth nonstationary linearization, and so a polynomial normal form is sought. Under the narrow band spectrum assumption, such forms were introduced in [GKt98, G02]. The assumption is satisfied, for example, by perturbations of algebraic systems. It ensures that the polynomial maps involved belong to a finite dimensional Lie group, which is important for applications. In GKt98, G02 Katok and Guysinsky proved existence of normal form coordinates $\left\{\mathcal{H}_{x}\right\}$ as well as a centralizer theorem. This was sufficient for some applications, such as local rigidity of higher rank actions [KtSp97]. However, smooth dependence of $\mathcal{H}_{x}$ along the leaves of $W$ was obtained under a strong extra assumption that the splitting of $T W$ into the spectral subspaces is smooth along $W$, rather than just continuous. This assumption is not typically satisfied by perturbations of algebraic systems. There were also no results on the coherence of maps $\mathcal{H}_{x}$ on the leaves of $W$. A geometric point of view on normal forms was developed by Feres in [Fe04], where he established existence of an $f$-invariant infinitesimal structure. If this structure, a certain generalized connection, is smooth then it can be integrated to recover normal forms. The smoothness, however, again relied on the smoothness of the spectral splitting.

In this paper we overcome these difficulties and obtain a system of normal form coordinates $\left\{\mathcal{H}_{x}\right\}$ which are smooth along the leaves of $W$ and on each leaf give a coherent atlas with transition maps in a finite dimensional Lie group. This gives homogeneous space structures on the leaves of $W$ which are invariant under $f$. Our results hold for any narrow band spectrum system without any extra assumptions. In particular, they apply to perturbations of algebraic systems. We also note that, in 
contrast to the case of the non-stationary linearization, the system $\left\{\mathcal{H}_{x}\right\}$ is not unique. In fact, the construction in GKt98, G02 produces $\mathcal{H}_{x}$ which may not be smooth if the spectral splitting in not. We give a modified construction that allows us to obtain the desired properties of $\mathcal{H}_{x}$.

\section{Definitions and Results}

Let $\mathcal{M}$ be a smooth compact connected manifold and let $f$ be a $C^{\infty}$ diffeomorphism of $\mathcal{M}$. Let $W$ be an $f$-invariant topological foliation of $\mathcal{M}$ with uniformly $C^{\infty}$ leaves. The latter means that all leaves are $C^{\infty}$ submanifolds and that all their derivatives are also continuous transversally to the leaves. Slightly more generally, we can consider a homeomorphism $f$ which is uniformly smooth along the leaves of $W$. We assume that $f$ contracts $W$, i.e. $\left\|\left.D f\right|_{T W}\right\|<1$ in some metric.

Let $\mathcal{E}=T W$ be the tangent bundle of the foliation $W$. We denote by $F$ the automorphism of $\mathcal{E}$ given by the derivative of $f$ :

$$
F_{x}=\left.D f\right|_{T_{x} W}: \mathcal{E}_{x} \rightarrow \mathcal{E}_{f x} .
$$

$F$ induces a bounded linear operator $F^{*}$ on the space of continuous sections of $\mathcal{E}$ by $F^{*} v(x)=F\left(v\left(f^{-1} x\right)\right)$. The spectrum of complexification of $F^{*}$ is called Mather spectrum of $F$. Under a mild assumption that non-periodic points of $f$ are dense in $\mathcal{M}$, Mather spectrum consists of finitely many closed annuli centered at 0 , see e.g. $[\mathrm{P}]$.

Definition 2.1. We say that $F$ has narrow band spectrum if its Mather spectrum is contained in a finite union of closed annuli $A_{i}, i=1, \ldots, l$, bounded by circles of radii $e^{\lambda_{i}}$ and $e^{\mu_{i}}$, where the numbers $\lambda_{1} \leq \mu_{1}<\cdots<\lambda_{l} \leq \mu_{l}<0$ satisfy

$$
\mu_{i}+\mu_{l}<\lambda_{i} \text { for } i=1, \ldots, l .
$$

This condition can be written as $\mu_{i}-\lambda_{i}<-\mu_{l}$ for $i=1, \ldots, l$, so it means that the length of each of the intervals $\left[\lambda_{i}, \mu_{i}\right]$ is smaller than that of $\left[\mu_{l}, 0\right]$. When there is only one spectral interval, (2.1) is the uniform $1 / 2$ pinching condition which yields non-stationary linearizations and was used in [Fe95] to construct an invariant affine connection.

For the given spectral intervals $\left\{\left[\lambda_{i}, \mu_{i}\right]\right\}$, the bundle $\mathcal{E}$ splits into a direct sum

$$
\mathcal{E}=\mathcal{E}^{1} \oplus \cdots \oplus \mathcal{E}^{l}
$$

of continuous $F$-invariant sub-bundles so that Mather spectrum of $\left.F\right|_{\mathcal{E}^{i}}$ is contained in the annulus $A_{i}$. This can be expressed using a convenient metric GKt98]: for each $i=1, \ldots, l$ and each $\varepsilon>0$ there exists a continuous metric $\|.\|_{x, \varepsilon}$ on $\mathcal{E}^{i}$ such that

$$
e^{\lambda_{i}-\varepsilon}\|t\|_{x, \varepsilon} \leq\left\|F_{x}(t)\right\|_{f x, \varepsilon} \leq e^{\mu_{i}+\varepsilon}\|t\|_{x, \varepsilon} \text { for every } t \in \mathcal{E}_{x}^{i} .
$$

Definition 2.2. A sub-resonance relation for $(\lambda, \mu)=\left(\lambda_{1}, \ldots, \lambda_{l}, \mu_{1}, \ldots, \mu_{l}\right)$ with $\lambda_{1} \leq \mu_{1}<\cdots<\lambda_{l} \leq \mu_{l}<0$ is a relation of the form

$$
\lambda_{i} \leq \sum s_{j} \mu_{j}, \quad \text { where } s_{1}, \ldots, s_{l} \text { are non-negative integers. }
$$


Clearly, $s_{j}=0$ for $j<i$, and $\sum s_{j} \leq \lambda_{1} / \mu_{l}$. The narrow band condition (2.1) implies that if $s_{i} \neq 0$, then $s_{i}=1$ and $s_{j}=0$ for $j>i$, and hence all sub-resonance relations are of the form $\lambda_{i} \leq \mu_{i}$ or $\lambda_{i} \leq \sum_{j \geq i+1} s_{j} \mu_{j}$.

For any vector spaces $E$ and $\bar{E}$ we say that a map $P: E \rightarrow \bar{E}$ is polynomial if for some, and hence every, bases of $E$ and $\bar{E}$ each component of $P$ is a polynomial. A polynomial map $P$ is homogeneous of degree $n$ if $P(a v)=a^{n} P(v)$ for all $v \in E$ and $a \in \mathbb{R}$. More generally, for a given splitting $E=E^{1} \oplus \cdots \oplus E^{l}$ we say that $P: E \rightarrow \bar{E}$ has homogeneous type $s=\left(s_{1}, \ldots, s_{l}\right)$ if for any real numbers $a_{1}, \ldots, a_{l}$ and vectors $t_{j} \in E^{j}, j=1, \ldots, l$, we have

$$
P\left(a_{1} t_{1}+\cdots+a_{l} t_{l}\right)=a_{1}^{s_{1}} \cdot \ldots \cdot a_{l}^{s_{l}} P\left(t_{1}+\cdots+t_{l}\right) .
$$

Any polynomial map can be written uniquely as a linear combination of terms of specific homogeneous types.

Definition 2.3. Suppose $E=E^{1} \oplus \cdots \oplus E^{l}, \bar{E}=\bar{E}^{1} \oplus \cdots \oplus \bar{E}^{l}$ and $P: E \rightarrow \bar{E}$ is a polynomial map. We split $P$ into components $P_{i}: E \rightarrow \bar{E}^{i}, P=\left(P_{1}, \ldots, P_{l}\right)$. We say that $P$ is of $(\lambda, \mu)$ sub-resonance type if each component $P_{i}$ has only terms of homogeneous types $s=\left(s_{1}, \ldots, s_{l}\right)$ satisfying sub-resonance relations $\lambda_{i} \leq \sum s_{j} \mu_{j}$.

We denote by $\mathcal{S}^{\lambda, \mu}(E, \bar{E})$ the space of all polynomials $E \rightarrow \bar{E}$ of $(\lambda, \mu)$ sub-resonance type.

It follows from the definition that polynomials in $\mathcal{S}^{\lambda, \mu}(E, \bar{E})$ have degree at most

$$
d=d(\lambda, \mu)=\left\lfloor\lambda_{1} / \mu_{l}\right\rfloor .
$$

It was shown in GKt98 that in the case of point spectrum, that is $\lambda_{i}=\mu_{i}$ for $i=$ $1, \ldots, l$, the elements of $\mathcal{S}^{\lambda, \lambda}(E, E)$ with $P(0)=0$ and invertible derivative at the origin form a group $G^{\lambda, \lambda}(E)$ with respect to composition. More generally, if $(\lambda, \mu)$ satisfies the narrow band condition, they generate (under composition) a finite-dimensional Lie group which we denote by $G^{\lambda, \mu}(E)$. The maps in $G^{\lambda, \mu}$ are called sub-resonance generated and can be described by adding finitely many relations to the set of subresonance ones. In fact, $G^{\lambda, \mu}(E)$ is contained in $G^{\lambda^{\prime}, \lambda^{\prime}}(E)$ for a certain $\lambda^{\prime}$, explicitly written in terms of $(\lambda, \mu)$ GKt98. This larger group may be used in place of $G^{\lambda, \mu}(E)$, for simplicity, in all arguments.

We return to our setting with fixed $F, \mathcal{E}$, and $(\lambda, \mu)$. We denote by $\mathcal{S}_{x, y}=S^{\lambda, \mu}\left(\mathcal{E}_{x}, \mathcal{E}_{y}\right)$ the space of sub-resonance polynomials and by $G_{x}=G^{\lambda, \mu}\left(\mathcal{E}_{x}\right)$ the group of subresonance generated polynomial maps. For any $x$ and $y$ in $\mathcal{M}$, any invertible linear map $A: \mathcal{E}_{y} \rightarrow \mathcal{E}_{x}$ that respects the splitting induces an isomorphism between the groups $G_{x}$ and $G_{y}$. The set $G_{x, y}$ of invertible sub-resonance generated polynomial maps from $\mathcal{E}_{x}$ to $\mathcal{E}_{y}$ is also naturally defined either by specifying homogeneity types using relations or simply by identifying $\mathcal{E}_{x}$ with $\mathcal{E}_{y}: G_{x, y}=\left\{P: \mathcal{E}_{x} \rightarrow \mathcal{E}_{y} \mid A \circ P \in G_{x}\right\}$ for any $A$ as above. Of course, if the bundle $\mathcal{E}$ and the sub-bundles $\mathcal{E}^{i}$ are trivial, then all $G_{x}$ and $G_{x, y}$ can be identified with a single group $G^{\lambda, \mu}(E)$. 
Theorem 2.4. Let $\mathcal{M}$ be a smooth compact connected manifold and let $f$ be a $C^{\infty}$ diffeomorphism of $\mathcal{M}$. Let $W$ be an $f$-invariant topological foliation of $\mathcal{M}$ with uniformly $C^{\infty}$ leaves. Suppose that $W$ is contracted by $f$, i.e. $\left\|\left.D f\right|_{T W}\right\|<1$ for some metric, and that $\left.D f\right|_{T W}$ has narrow band spectrum, see Definition [2.1.

Then there exist a family $\left\{\mathcal{H}_{x}\right\}_{x \in \mathcal{M}}$ of $C^{\infty}$ diffeomorphisms

$$
\mathcal{H}_{x}: W_{x} \rightarrow \mathcal{E}_{x}=T_{x} W \quad \text { such that }
$$

(i) $\mathcal{P}_{x}=\mathcal{H}_{f x} \circ f \circ \mathcal{H}_{x}^{-1}: \mathcal{E}_{x} \rightarrow \mathcal{E}_{f x}$ is a polynomial map of sub-resonance type for each $x \in \mathcal{M}$,

(ii) $\mathcal{H}_{x}(x)=0$ and $D_{x} \mathcal{H}_{x}$ is the identity map for each $x \in \mathcal{M}$,

(iii) $\mathcal{H}_{x}$ depends continuously on $x \in \mathcal{M}$ in $C^{\infty}$ topology and it depends smoothly on $x$ along the leaves of $W$,

(iv) $\mathcal{H}_{y} \circ \mathcal{H}_{x}^{-1}: \mathcal{E}_{x} \rightarrow \mathcal{E}_{y}$ is a sub-resonance generated polynomial for each $x \in \mathcal{M}$ and each $y \in W_{x}$.

Another way to interpret (iv) is to view $\mathcal{H}_{x}$ as a coordinate chart on $W_{x}$, identifying it with $\mathcal{E}_{x}$, see Section 3.2 for more details. In this coordinate chart, (iv) yields that all transition maps $\mathcal{H}_{y} \circ \mathcal{H}_{x}^{-1}$ for $y \in W_{x}$ are in $\bar{G}_{x}$, the group generated by $G_{x}$ and the translations of $\mathcal{E}_{x}$. Thus $\mathcal{H}_{x}$ gives the leaf a structure of homogeneous space $W_{x} \sim$ $\bar{G}_{x} / G_{x}$, which is consistent with other coordinate charts $\mathcal{H}_{y}$ for $y \in W_{x}$ and is preserved by the normal form $\mathcal{P}_{x}$ according to (i).

Remark 2.5. An important and useful feature of normal forms for contractions was established in GKt98]. Let $g$ be a homeomorphism of $\mathcal{M}$ which commutes with $f$, preserves $W$, and is smooth along the leaves of $W$. Then the maps $\mathcal{H}_{x}$ bring $g$ to a normal form as well, i.e. the map $Q_{x}=\mathcal{H}_{f x} \circ g \circ \mathcal{H}_{x}^{-1}$ is a sub-resonance generated polynomial for each $x \in \mathcal{M}$.

\section{Proof of Theorem 2.4}

3.1. Proof of (i), (ii), (iii). Let us fix a small tubular neighborhood $U$ of the zero section in $T W$ such that for each $x$ in $\mathcal{M}$, we can identify the open set $U_{x}=U \cap T_{x} W$ in $T_{x} W$ with a neighborhood of $x$ in the leaf $W_{x}$ using the exponential map. The size of this neighborhood can be chosen the same for all $x \in \mathcal{M}$. Thus we can view the diffeomorphism $f$ as a family of maps $\mathcal{F}_{x}=\left.f\right|_{U_{x}}$ from $U_{x} \subset T_{x} W$ to $U_{f x} \subset T_{f x} W$. Our first goal is to obtain a formal power series for $\mathcal{H}_{x}$.

For each map $\mathcal{F}_{x}: \mathcal{E}_{x} \rightarrow \mathcal{E}_{f x}$ we consider its formal power series at $t=0$ :

$$
\mathcal{F}_{x}(t)=\sum_{n=1}^{\infty} F_{x}^{(n)}(t) .
$$

As a function of $t$ for a fixed $x, F_{x}^{(n)}(t): \mathcal{E}_{x} \rightarrow \mathcal{E}_{f x}$ is a vector valued homogeneous polynomial map of degree $n$ (that is, in any bases of $\mathcal{E}_{x}$ and $\mathcal{E}_{f x}$, each coordinate in $\mathcal{E}_{f x}$ is a homogeneous polynomial of degree $n$ of the coordinates in $\mathcal{E}_{x}$ ). 
First we construct the formal power series at $t=0$ for the desired coordinate change $\mathcal{H}_{x}(t)$ as well as the finite power series at $t=0$ for the resulting polynomial extension $\mathcal{P}_{x}(t)$. We use notations for these series similar to (3.1):

$$
\mathcal{H}_{x}(t)=\sum_{n=1}^{\infty} H_{x}^{(n)}(t) \quad \text { and } \quad \mathcal{P}_{x}(t)=\sum_{n=1}^{d} P_{x}^{(n)}(t) .
$$

We will use notation $F_{x}=F_{x}^{(1)}$ for the first derivative of $\mathcal{F}$. For $\mathcal{H}$ and $\mathcal{P}$ we choose

$$
H_{x}^{(1)}=\mathrm{Id}: \mathcal{E}_{x} \rightarrow \mathcal{E}_{x} \quad \text { and } \quad P_{x}^{(1)}=F_{x} \quad \text { for all } x \in \mathcal{M} .
$$

We construct the terms $H_{x}^{(n)}$ inductively to "eliminate" the part of $F_{x}^{(n)}$ which is not of sub-resonance type. More precisely, we ensure that the terms $P_{x}^{(n)}$ determined by the conjugacy equation are of sub-resonance type. The base of the induction is the linear terms chosen above. We will obtain $H_{x}^{(n)}$ and $F_{x}^{(n)}$ which are continuous in $x$ on $\mathcal{M}$ and smooth in $x$ along the leaves of $W$.

Using the notations above we can write the conjugacy equation

$$
\mathcal{H}_{f x} \circ \mathcal{F}_{x}=\mathcal{P}_{x} \circ \mathcal{H}_{x}
$$

as follows:

$$
\left(\mathrm{Id}+\sum_{k=2}^{\infty} H_{f x}^{(k)}\right) \circ\left(F_{x}+\sum_{k=2}^{\infty} F_{x}^{(k)}\right)=\left(F_{x}+\sum_{k=2}^{\infty} P_{x}^{(k)}\right) \circ\left(\mathrm{Id}+\sum_{k=2}^{\infty} H_{x}^{(k)}\right)
$$

for all $x \in \mathcal{M}$. Considering the terms of degree $n$, we obtain for $n=2$

$$
F_{x}^{(2)}+H_{f x}^{(2)} \circ F_{x}=F_{x} \circ H_{x}^{(2)}+P_{x}^{(2)},
$$

and in general for $n \geq 2$

$$
F_{x}^{(n)}+H_{f x}^{(n)} \circ F(x)+\sum H_{f x}^{(k)} \circ F_{x}^{(j)}=F_{x} \circ H_{x}^{(n)}+P_{x}^{(n)}+\sum P_{x}^{(j)} \circ H_{x}^{(k)},
$$

where the summations are over all $k$ and $j$ such that $k j=n$ and $1<k, j<n$. We rewrite the equation as

$$
F_{x}^{-1} \circ P_{x}^{(n)}=-H_{x}^{(n)}+F_{x}^{-1} \circ H_{f x}^{(n)} \circ F_{x}+Q_{x},
$$

where

$$
Q_{x}=F_{x}^{-1}\left(F_{x}^{(n)}+\sum_{k j=n, 1<k, j<n} H_{f x}^{(k)} \circ F_{x}^{(j)}-P_{x}^{(j)} \circ H_{x}^{(k)}\right) .
$$

We note that $Q_{x}$ is composed only of terms $H^{(k)}$ and $P^{(k)}$ with $1<k<n$, which are already constructed, and terms $F^{(k)}$ with $1<k \leq n$, which are given. Thus by the inductive assumption $Q_{x}$ is continuous in $x$ on $\mathcal{M}$ and smooth along the leaves of $W$.

We denote by $\mathcal{R}_{x}^{(n)}$ the vector space of all homogeneous polynomial maps of degree $n$ from $\mathcal{E}_{x}$ to $\mathcal{E}_{x}$. Identifying these polynomial maps with symmetric $n$-linear maps, one can view this space as $\operatorname{Sym}_{n}\left(\mathcal{E}_{x}^{*}\right) \otimes \mathcal{E}_{x}$, where the former is the space symmetric elements in the $n^{\text {th }}$ tensor power of the dual space of $\mathcal{E}_{x}$, see $\mathrm{Fe} 04$. Let $\mathcal{R}^{(n)}$ be the vector bundle 
over $\mathcal{M}$ whose fiber at $x$ is $\mathcal{R}_{x}^{(n)}$. We denote by $\mathcal{S}_{x}^{(n)}$ and $\mathcal{N}_{x}^{(n)}$ the subspaces of $\mathcal{R}_{x}^{(n)}$ consisting of sub-resonance and non sub-resonance polynomials respectively. These subspaces depend continuously on $x$ and thus $\mathcal{R}^{(n)}$ splits into the direct sum of the continuous sub-bundles $\mathcal{S}^{(n)} \oplus \mathcal{N}^{(n)}$.

Our goal is to find a section $H^{(n)}$ of $\mathcal{R}^{(n)}$ so that the right side of (3.2) is a section of $\mathcal{S}^{(n)}$, and hence so is $P^{(n)}$ when defined by this equation. The sub-bundle $\mathcal{N}^{(n)}$ in general is only continuous. To construct $H_{x}^{(n)}$ which depends smoothly on $x$ along the leaves of $W$ we will work with the factor bundle $\mathcal{R}^{(n)} / \mathcal{S}^{(n)}$ rather than with the splitting $\mathcal{S}^{(n)} \oplus \mathcal{N}^{(n)}$. The fiber of $\mathcal{R}^{(n)} / \mathcal{S}^{(n)}$ at $x$ is the vector space $\mathcal{R}_{x}^{(n)} / \mathcal{S}_{x}^{(n)}$ and, as a continuous vector bundle, $\mathcal{R}^{(n)} / \mathcal{S}^{(n)}$ is isomorphic to $\mathcal{N}^{(n)}$ via the natural identification. A local trivialization of $\mathcal{R}^{(n)} / \mathcal{S}^{(n)}$ can be obtained by fixing locally a constant transversal to $\mathcal{S}^{(n)}$ in any trivialization of $\mathcal{R}^{(n)}$. We will show in Lemma 3.3] below that the subspace $\mathcal{S}_{x}^{(n)}$ depends smoothly on $x$ along the leaves of $W$, and hence the bundle $\mathcal{R}^{(n)} / \mathcal{S}^{(n)}$ and the projection to it from $\mathcal{R}^{(n)}$ are smooth along the leaves of $W$.

Projecting (3.2) to the factor bundle $\mathcal{R}^{(n)} / \mathcal{S}^{(n)}$, our goal is to solve the equation

$$
0=-\bar{H}_{x}^{(n)}+F_{x}^{-1} \circ \bar{H}_{f x}^{(n)} \circ F_{x}+\bar{Q}_{x}
$$

where $\bar{H}^{(n)}$ and $\bar{Q}$ are the projections of $H^{(n)}$ and $Q$ respectively.

We consider the bundle automorphism $\Phi: \mathcal{R}^{(n)} \rightarrow \mathcal{R}^{(n)}$ covering $f^{-1}: \mathcal{M} \rightarrow \mathcal{M}$ given by the maps $\Phi_{x}: \mathcal{R}_{f x}^{(n)} \rightarrow \mathcal{R}_{x}^{(n)}$

$$
\Phi_{x}(R)=F_{x}^{-1} \circ R \circ F_{x} .
$$

Since $F$ preserves the splitting $\mathcal{E}=\mathcal{E}^{1} \oplus \cdots \oplus \mathcal{E}^{l}$, it follows from the definition that the sub-bundles $\mathcal{S}^{(n)}$ and $\mathcal{N}^{(n)}$ are $\Phi$-invariant. We denote by $\bar{\Phi}$ the induced automorphism of $\mathcal{R}^{(n)} / \mathcal{S}^{(n)}$ and conclude that (3.3) is equivalent to

$$
\bar{H}_{x}^{(n)}=\tilde{\Phi}_{x}\left(\bar{H}_{f x}^{(n)}\right), \quad \text { where } \tilde{\Phi}_{x}(R)=\bar{\Phi}_{x}(R)+\bar{Q}_{x} .
$$

Thus a solution of (3.3) is a $\tilde{\Phi}$-invariant section of $\mathcal{R}^{(n)} / \mathcal{S}^{(n)}$. Lemma 3.1 below shows that $\tilde{\Phi}$ is a contraction and hence has a unique continuous invariant section, which can be explicitly written as

$$
\bar{H}_{x}^{(n)}=\sum_{k=0}^{\infty}\left(F_{x}^{k}\right)^{-1} \circ \bar{Q}_{f^{k} x} \circ F_{x}^{k}, \quad \text { where } F_{x}^{k}=F_{f^{k-1} x} \circ \cdots \circ F_{f x} \circ F_{x} .
$$

To show that the maps $\bar{H}_{x}^{(n)}$ depend smoothy on $x$ along the leaves of $W$ we use the version of the $C^{r}$ Section Theorem of Hirsch, Pugh, and Shub formulated below. We apply the theorem with $\mathcal{B}=\mathcal{R}^{(n)} / \mathcal{S}^{(n)}$ and $\Psi=\tilde{\Phi}$. Since $f^{-1}$ expands $W$, we have $\alpha_{x}>1$, which yields that $\bar{H}_{x}$ is $C^{r}$ along the leaves of $W$ for any $r$. We conclude that (3.6) gives the unique solution of (3.3) which is smooth along the leaves of $W$. Now we take a lift $H^{(n)}$ of $\bar{H}^{(n)}$ to $\mathcal{R}^{(n)}$, which is not unique. We take a continuous sub-bundle $\tilde{\mathcal{N}}^{(n)}$ which is smooth along the leaves of $W$ so that subspace $\tilde{\mathcal{N}}_{x}^{(n)}$ is sufficiently close 
to $\mathcal{N}_{x}^{(n)}$ and hence is transverse to $\mathcal{S}_{x}^{(n)}$ for each $x \in \mathcal{M}$. Then we define $H_{x}^{(n)}$ as the unique element in $\tilde{\mathcal{N}}_{x}^{(n)}$ that projects to $\bar{H}_{x}^{(n)}$. This lift $H^{(n)}$ is smooth along the leaves of $W$ and it is the desired section of $\mathcal{R}^{(n)}$ so that the right side of (3.2) is section of $\mathcal{S}^{(n)}$. Finally, we define $P_{x}^{(n)}$ from $(\underline{3.2})$ and note that it is in $\mathcal{S}_{x, f x}^{(n)}$ and smooth along the leaves of $W$ since $H_{x}^{(n)}$ is.

$C^{r}$ Section Theorem [HPS]. Let $f$ be a $C^{r}, r \geq 1$, diffeomorphism of a compact smooth manifold $\mathcal{M}$. Let $W$ be an $f$-invariant topological foliation with uniformly $C^{r}$ leaves. Let $\mathcal{B}$ be a normed vector bundle over $\mathcal{M}$ and $\Psi: \mathcal{B} \rightarrow \mathcal{B}$ be an extension of $f$ such that both $\mathcal{B}$ and $\Psi$ are uniformly $C^{r}$ along the leaves of $W$.

Suppose that $\Psi$ contracts fibers of $\mathcal{B}$, i.e. for any $x \in \mathcal{M}$ and any $u, w \in \mathcal{B}(x)$

$$
\|\Psi(u)-\Psi(w)\|_{f x} \leq k_{x}\|u-w\|_{x} \text { with } \sup \left\{k_{x}: x \in \mathcal{M}\right\}<1 .
$$

Then there exists a unique continuous $\Psi$-invariant section of $\mathcal{B}$. Moreover, if

$$
\sup \left\{k_{x} \alpha_{x}^{r}: x \in \mathcal{M}\right\}<1 \text {, where } \alpha_{x}=\left\|\left(\left.D f\right|_{T_{x} W}\right)^{-1}\right\|,
$$

then the unique invariant section is uniformly $C^{r}$ smooth along the leaves of $W$.

This version of the $C^{r}$ Section Theorem (see [KS07, Theorem 3.7]) summarizes for our context Theorems 3.1, 3.2, and 3.5, and Remarks 1 and 2 after Theorem 3.8 in [HPS]. In this theorem the smoothness of the invariant section is obtained only along $W$, so the smoothness of $\mathcal{B}$ and $\Psi$ is required along $W$ only. It also follows from the proof in [HPS] that the contraction in the base (3.8) needs to be estimated only along $W$ (formally, this can be obtained by applying the theorem with the base manifold $\mathcal{M}$ considered as the disjoint union of the leaves of $W$ ). This has been observed in the study of partially hyperbolic systems, see for example Introduction, Theorem 3.1 and remarks in [PSW97].

Lemma 3.1. The map $\Phi: \mathcal{N}^{(n)} \rightarrow \mathcal{N}^{(n)}$ given by (3.4) is a contraction in the sense of (3.7), and hence so is $\tilde{\Phi}: \mathcal{R}^{(n)} / \mathcal{S}^{(n)} \rightarrow \mathcal{R}^{(n)} / \mathcal{S}^{(n)}$ given by (3.5).

Proof. We recall that the norm of a homogeneous polynomial map $R: E \rightarrow \bar{E}$ of degree $n$ is defined as $\|R\|=\sup \{\|R(v)\|: v \in E,\|v\|=1\}$. If $P$ is a linear or a homogeneous polynomial map $\tilde{E} \rightarrow E$, then for the norm of the composition we have

$$
\|R \circ P\| \leq\|R\| \cdot\|P\|^{n} .
$$

Suppose that $E=E^{1} \oplus \ldots \oplus E^{l}$ and $\|v\|=\max _{i}\left\|v_{i}\right\|$ for $v=\left(v_{1}, \ldots, v_{l}\right)$. If $R$ is of homogeneous type $s=\left(s_{1}, \ldots, s_{l}\right)$ then by (2.5) we get $\|R(v)\| \leq\|R\|\left\|v_{1}\right\|^{s_{1}} \ldots\left\|v_{l}\right\|^{s_{l}}$.

We will use the norm on $\mathcal{E}$ which is the maximum of the norms on $\mathcal{E}^{i}$ used in (2.3): $\|t\|=\|t\|_{x, \varepsilon}=\max _{i}\left\|t_{i}\right\|_{x, \varepsilon}$ for any $t=\left(t_{1}, \ldots, t_{l}\right) \in \mathcal{E}_{x}$.

Then for a polynomial $R: \mathcal{E}_{f x} \rightarrow \mathcal{E}_{f x}^{i}$ of homogeneous type $s=\left(s_{1}, \ldots, s_{l}\right)$ we have

$$
\left\|R \circ F_{x}\right\| \leq\|R\| \cdot\left\|\left.F\right|_{\mathcal{E}_{x}^{1}}\right\|^{s_{1}} \ldots\left\|\left.F\right|_{\mathcal{E}_{x}^{l}}\right\|^{s_{l}}
$$


If $R \in \mathcal{N}_{f x}$ then, by definition, we have $\lambda_{i}>\sum s_{j} \mu_{j}$. We can choose a sufficiently small $\varepsilon>0$ so that $\lambda_{i}>\sum s_{j} \mu_{j}+(n+2) \varepsilon$ for all such relations. It follows from (2.3) that $\left\|\left.F\right|_{\mathcal{E}_{x}^{j}}\right\| \leq e^{\mu_{j}+\varepsilon}$ and $\left\|\left.F\right|_{\mathcal{E}_{x}^{i}} ^{-1}\right\| \leq e^{-\lambda_{i}+\varepsilon}$, so we conclude that for each $x \in \mathcal{M}$

$$
\begin{aligned}
\left\|\Phi_{x}(R)\right\| & \leq\left\|\left.F\right|_{\mathcal{E}_{x}^{i}} ^{-1}\right\| \cdot\|R\| \cdot \prod_{j}\left\|\left.F\right|_{\mathcal{E}_{x}^{j}}\right\|^{s_{j}} \leq e^{-\lambda_{i}+\varepsilon} \cdot\|R\| \cdot \prod_{j}\left(e^{\mu_{j}+\varepsilon}\right)^{s_{j}} \leq \\
& \leq e^{-\lambda_{i}+\sum s_{j} \mu_{j}+(n+1) \varepsilon} \cdot\|R\| \leq e^{-\varepsilon}\|R\| .
\end{aligned}
$$

Thus $\Phi$ is a contraction on $\mathcal{N}^{(n)}$. The second statement follows since the linear part $\bar{\Phi}$ of $\tilde{\Phi}$ is a contraction. Indeed, $\bar{\Phi}$ corresponds to $\Phi$ under the identification of $\mathcal{R}^{(n)} / \mathcal{S}^{(n)}$ and $\mathcal{N}^{(n)}$ as continuous vector bundles given by the natural linear isomorphisms between $\mathcal{R}_{x}^{(n)} / \mathcal{S}_{x}^{(n)}$ and $\mathcal{N}_{x}^{(n)}$, which depend continuously on the base point. Hence $\left\|\bar{\Phi}_{x}\right\| \leq e^{-\varepsilon}$ with respect to a continuous family of norms on the fibers of $\mathcal{R}^{(n)} / \mathcal{S}^{(n)}$.

Proposition 3.2. The vector space of sub-resonance polynomials $\mathcal{S}(E)=\mathcal{S}^{(\lambda, \mu)}(E, E)$ for the given $(\lambda, \mu)$ and splitting $E=E^{1} \oplus \cdots \oplus E^{l}$ depends only on the fast flag $V$ :

$$
E^{1}=V^{1} \subset V^{2} \subset \ldots \subset V^{l}=E, \quad \text { where } V^{i}=E^{1} \oplus \cdots \oplus E^{i} .
$$

More precisely, $\mathcal{S}(E)=\mathcal{S}(\tilde{E})$ if $\tilde{E}$ is $E$ equipped with a splitting that generates the same fast flag: $V^{i}=\tilde{E}^{1} \oplus \cdots \oplus \tilde{E}^{i}$, for $i=1, \ldots, l$.

Proof. Let $A: E \rightarrow \tilde{E}$ be a linear isomorphism with $A\left(E^{i}\right)=\tilde{E}^{i}$, for $i=1, \ldots, l$. Then $R \in \mathcal{S}(E)$ if and only if $\tilde{R}=A \circ R \circ A^{-1} \in \mathcal{S}(\tilde{E})$. Also, $A$ and $A^{-1}$ are block triangular for the splitting $E=E^{1} \oplus \cdots \oplus E^{l}$, that is their matrices are block triangular in any basis adapted to this splitting or, equivalently, they belong to $\mathcal{S}(E)$. To complete the proof we need to show that if $R \in \mathcal{S}(E)$ then so are $A \circ R$ and $R \circ A^{-1}$.

By splitting $R$ into components and homogeneity types, it suffices to check this for $R$ : $E \rightarrow E^{i}$ corresponding to a sub-resonance relation $\lambda_{i} \leq \sum s_{j} \mu_{j}$, whose combinatorial type we dente by $\left(i ; s_{1}, \ldots, s_{l}\right)$. We call a relation $\left(m ; s_{1}^{\prime}, \ldots, s_{l}^{\prime}\right)$ with $\left|s^{\prime}\right|=|s|$ subordinate to $\left(i ; s_{1}, \ldots, s_{l}\right)$ if $m \leq i$ and $\sum_{j \leq k} s_{j}^{\prime} \leq \sum_{j \leq k} s_{j}$ for each $k$ (this gives a partial order on relations). It follows that $\lambda_{m} \leq \sum s_{j}^{\prime} \mu_{j}$ so that all subordinate relations are also of sub-resonance type. It is easy to see that the homogeneity type of any term in $A \circ R$ or $R \circ A^{-1}$ corresponds to a relation subordinate to $\left(i ; s_{1}, \ldots, s_{l}\right)$, and hence these polynomials are in $\mathcal{S}(E)$.

In fact, the subspace spanned by all sub-resonance polynomials corresponding to the relations subordinate to $\left(i ; s_{1}, \ldots, s_{l}\right)$ can be explicitly described in terms of the flag $V$ only: it consists of all polynomial maps $R: \mathcal{E}_{x} \rightarrow V_{x}^{i}$ such that for each $k$ the degree of $R$ along the subspace $V_{x}^{k}$ is at most $\sum_{j \leq k} s_{j}$. This is an alternative way to see that $\mathcal{S}(E)$ depends only on the fast flag.

Lemma 3.3. The fibers $\mathcal{S}_{x}^{(n)}$ of the vector sub-bundle $\mathcal{S}^{(n)} \subset \mathcal{R}^{(n)}$ depend smoothly on $x$ along the leaves of $W$. 
Proof. We consider the fast flag $\mathcal{V}_{x}$ in $\mathcal{E}_{x}$ :

$$
\mathcal{E}_{x}^{1}=\mathcal{V}_{x}^{1} \subset \mathcal{V}_{x}^{2} \subset \ldots \subset \mathcal{V}_{x}^{l}=\mathcal{E}_{x}, \quad \text { where } \mathcal{V}_{x}^{i}=\mathcal{E}_{x}^{1} \oplus \cdots \oplus \mathcal{E}_{x}^{i}
$$

First we note that the subspaces $\mathcal{V}_{x}^{i}$ depend smoothly on $x$ along $W$. This is relatively well-known and is proved by an application of the $C^{r}$ Section Theorem to a suitable graph transform extension, see [KS07, Proposition 3.9] for an explicit reference. In fact, in our setting, smoothness of the fast flag along $W$ follows directly from normal forms since $\mathcal{H}_{x}$ maps the fast sub-foliations of $W$ to linear foliations of $\mathcal{E}_{x}$, see Section 3.2 for details (smooth dependence of $\mathcal{H}_{x}$ on $x$ is not needed for this argument, so there is no circular reasoning).

It now follows that there exist a splitting $\mathcal{E}_{x}=\tilde{\mathcal{E}}_{x}^{1} \oplus \cdots \oplus \tilde{\mathcal{E}}_{x}^{l}$ which is smooth in $x$ along $W$ and defines the same flag $\mathcal{V}_{x}$. Then Proposition 3.2 implies that $\mathcal{S}_{x}^{(n)}=\tilde{\mathcal{S}}_{x}^{(n)}$, the space of all sub-resonance homogeneous polynomials of degree $n$ for the same $(\lambda, \mu)$ and the new splitting. The latter clearly depends smoothly on $x$ along $W$.

Thus we have constructed the formal series $\mathcal{H}_{x}(t)=\sum_{n=1}^{\infty} H_{x}^{(n)}(t)$ for the coordinate change and the polynomial map $\mathcal{P}_{x}(t)=\sum_{n=1}^{d} P_{x}^{(n)}(t)$, where $d=\left\lfloor\lambda_{1} / \mu_{l}\right\rfloor$. Now we obtain the actual coordinate change function. We fix $N>d$ and conjugate $\mathcal{F}$ by the polynomial coordinate change $\overline{\mathcal{H}}^{N}$ given by

$$
\overline{\mathcal{H}}_{x}^{N}(t)=\sum_{n=1}^{N} H_{x}^{(n)}(t) \text { and denote } \tilde{\mathcal{F}}_{x}(t)=\overline{\mathcal{H}}_{f x}^{N} \circ \mathcal{F}_{x} \circ\left(\overline{\mathcal{H}}_{x}^{N}\right)^{-1} .
$$

We note that $\overline{\mathcal{H}}_{x}^{N}(t)$ is a diffeomorphism on a neighborhood $\tilde{U}_{x} \subset U_{x}$ of $0 \in \mathcal{E}_{x}$ since its differential at 0 is Id, moreover the size of $\tilde{U}_{x}$ can be bounded away from 0 by compactness of $\mathcal{M}$. By the construction of $\overline{\mathcal{H}}^{N}$, the maps $\tilde{\mathcal{F}}_{x}$ and $\mathcal{P}_{x}$ have the same derivatives at $t=0$ up to order $N$ for each $x \in \mathcal{M}$. We now look for the coordinate change conjugating $\tilde{\mathcal{F}}_{x}$ and $\mathcal{P}_{x}$ in the space

$$
\mathcal{C}_{x}=\left\{R_{x} \in C^{N}\left(\tilde{U}_{x}, \mathcal{E}_{x}\right): R_{x}(0)=0, D_{0} R_{x}=\mathrm{Id}, D_{0}^{(k)} R_{x}=0, k=2, \ldots, N\right\} .
$$

It is a closed affine subspace of $C^{N}\left(\tilde{U}_{x}, \mathcal{E}_{x}\right)$ with a standard norm

$$
\|R\|_{N}=\max \left\{\left\|D_{t}^{(k)} R\right\|: t \in U_{x}, 0 \leq k \leq N\right\} .
$$

Each element in $\mathcal{C}_{x}$ is a diffeomorphism on a neighborhood of $0 \in \mathcal{E}_{x}$. We will fix $\delta<1$ and then choose $\tilde{U}_{x}$ to be convex and small enough so that for any $R_{1}, R_{2}$ in $\mathcal{C}_{x}$ and $R=R_{1}-R_{2}$ we have

$$
\left\|D^{(k)} R\right\|_{0} \leq \delta\left\|D^{(N)} R\right\|_{0} \quad \text { for } 0 \leq k<N \quad \text { and hence } \quad\|R\|_{N}=\left\|R^{(N)}\right\|_{0} .
$$

This is possible since $D_{0}^{(k)} R=0$ for $0 \leq k \leq N$ and hence for any $t \in \tilde{U}_{x}$ and $0 \leq k<N$ we can estimate

$$
\left\|D_{t}^{(k)} R\right\| \leq\|t\| \cdot \sup \left\{\left\|D_{s}^{(k+1)} R\right\|:\|s\| \leq\|t\|\right\} \leq \operatorname{diam} \tilde{U}_{x} \cdot\left\|R^{(k+1)}\right\|_{0} .
$$


We consider the space $\mathcal{S}$ of continuous sections of the bundle $\mathcal{C}$ and equip it with the distance $\operatorname{dist}\left(R_{1}, R_{2}\right)=\left\|R_{1}-R_{2}\right\|_{N}=\sup _{x}\left\|\left(R_{1}-R_{2}\right)_{x}\right\|_{N}$. We consider the operator

$$
T[R]_{x}=\left(\mathcal{P}_{x}\right)^{-1} \circ R_{f x} \circ \tilde{\mathcal{F}}_{x}
$$

on $\mathcal{S}$ so that the fixed point of $T$ is the desired coordinate change. Note that $T[R]$ is in $\mathcal{S}$ by the definition of $\mathcal{C}_{x}$ and the coincidence of the derivatives of $\mathcal{P}$ and $\tilde{\mathcal{F}}$ at 0 .

To show that $T$ is a contraction on $\mathcal{S}$ we denote $R=R_{1}-R_{2}$ for $R_{1}, R_{2}$ in $\mathcal{S}$ and estimate $\|T[R]\|_{N}=\sup _{x}\left\|T[R]_{x}\right\|_{N}$, which by (3.13) equals to $\sup _{x}\left\|T[R]_{x}^{(N)}\right\|_{0}$.

$$
\begin{aligned}
D_{t}^{(N)} T[R]_{x} & =D_{t}^{(N)}\left(\left(\mathcal{P}_{x}\right)^{-1} \circ R_{f x} \circ \tilde{\mathcal{F}}_{x}\right)= \\
& =D_{R_{f x}\left(\tilde{\mathcal{F}}_{x}(t)\right)}^{(1)}\left(\mathcal{P}_{x}\right)^{-1} \circ D_{\tilde{\mathcal{F}}_{x}(t)}^{(N)} R_{f x} \circ D_{t}^{(1)} \tilde{\mathcal{F}}_{x}+J,
\end{aligned}
$$

where $J$ consists of a fixed number of terms of the type

$$
D_{R_{f x}\left(\tilde{\mathcal{F}}_{x}(t)\right)}^{(i)}\left(\mathcal{P}_{x}\right)^{-1} \circ D_{\tilde{\mathcal{F}}_{x}(t)}^{(j)} R_{f x} \circ D_{t}^{(k)} \tilde{\mathcal{F}}_{x}, \quad i j k=N, j<N
$$

whose norm can be estimated using (3.9) as

$$
\left\|D_{R_{f x}\left(\tilde{\mathcal{F}}_{x}(t)\right)}^{(i)}\left(\mathcal{P}_{x}\right)^{-1}\right\| \cdot\left\|D_{\tilde{\mathcal{F}}_{x}(t)}^{(j)} R_{f x}\right\|^{i} \cdot\left\|D_{t}^{(k)} \tilde{\mathcal{F}}_{x}\right\|^{i j} .
$$

By (3.13), $\left\|D_{\tilde{\mathcal{F}}_{x}(t)}^{(j)} R_{f x}\right\| \leq \delta\left\|D^{(N)} R_{f x}\right\|_{0}<1$ if $\delta$ is small enough. Therefore, there exists a constant $M=M(\mathcal{F}, \mathcal{P}, N)$ such that

$$
\|J\| \leq M \cdot \delta\left\|D^{(N)} R_{f x}\right\|_{0} \leq M \delta \cdot\|R\|_{N} .
$$

We estimate the main term in (3.14) as follows

$$
\begin{aligned}
& \left\|D_{R_{f x}\left(\tilde{\mathcal{F}}_{x}(t)\right)}^{(1)}\left(\mathcal{P}_{x}\right)^{-1} \circ D_{\tilde{\mathcal{F}}_{x}(t)}^{(N)} R_{f x} \circ D_{t}^{(1)} \tilde{\mathcal{F}}_{x}\right\| \leq \\
& \leq\left\|D_{R_{f x}\left(\tilde{\mathcal{F}}_{x}(t)\right)}^{(1)}\left(\mathcal{P}_{x}\right)^{-1}\right\| \cdot\|R\|_{N} \cdot\left\|D_{t}^{(1)} \tilde{\mathcal{F}}_{x}\right\|^{N} \leq \\
& \leq e^{-\lambda_{1}+\varepsilon} \cdot\|R\|_{N} \cdot e^{N \mu_{l}+N \varepsilon}=k^{\prime} \cdot\|R\|_{N},
\end{aligned}
$$

where $k^{\prime}=\exp \left(\left(N \mu_{l}-\lambda_{1}\right)+(N+1) \varepsilon\right)$. Since $\lambda_{1}>N \mu_{l}$ by the choice of $N$ we can take $\varepsilon$ small enough so that $k^{\prime}<1$. Combining the estimates (3.15) and (3.16) we get

$$
\left\|T\left[R_{1}-R_{2}\right]\right\|_{N}=\sup _{x}\left\|T\left[R_{1}-R_{2}\right]_{x}^{(N)}\right\|_{0} \leq\left(k^{\prime}+M \delta\right) \cdot\left\|R_{1}-R_{2}\right\|_{N}
$$

so that $T$ is a contraction if $\delta$ is small enough. Thus $T$ has a unique fixed point, which is a continuous family $\tilde{\mathcal{H}}_{x}^{N}$ of coordinate changes conjugating $\mathcal{P}_{x}$ and $\tilde{\mathcal{F}}_{x}$ given by (3.12).

We conclude that the maps $\mathcal{H}_{x}^{N}=\tilde{\mathcal{H}}_{x}^{N} \circ \overline{\mathcal{H}}_{x}^{N}$ give a family of $C^{N}$ diffeomorphisms defined on a neighborhood of the zero section of $\mathcal{E}$ which depend continuously on $x \in \mathcal{M}$ in $C^{N}$ topology and satisfy (i) and (ii) of Theorem 2.4. Since $f$ contracts $W$, this family extends uniquely to a family of $C^{N}$ global diffeomorphisms $\mathcal{H}_{x}^{N}: W_{x} \rightarrow \mathcal{E}_{x}$ that satisfy (i). Once we fix a formal power series for $\mathcal{H}$, the construction works for each $N>d$ and $\mathcal{H}^{N}$ is unique among $C^{N}$ diffeomorphisms whose derivatives up to order $N$ are given by the series. This means that all $\mathcal{H}^{N}$ coincide and give a family of $C^{\infty}$ diffeomorphisms. 
Thus we have proved parts (i),(ii) and (iii) with smoothness along $W$ established so far for the Taylor coefficients of $\mathcal{H}_{x}$ at 0 . The smoothness of $\mathcal{H}_{x}$ in $x$ along $W$ will follow from this once we establish part (iv). Indeed, in the coordinates on $W_{x}$ given by $\mathcal{H}_{x}$ for a fixed $x$, for all other $y \in W_{x}$ the maps $\mathcal{H}_{y}$ are polynomials and thus coincide with their finite Taylor series.

3.2. Consistency of the fast foliations. First we describe some properties of the fast flag (3.11) and related properties of $\mathcal{H}_{x}$ and of sub-resonance generated maps. The leaves of $W$ are subfoliated by unique foliations $U^{k}$ tangent to $V_{x}^{k}=\mathcal{E}_{x}^{1} \oplus \cdots \oplus \mathcal{E}_{x}^{k}$. We denote by $\bar{U}^{k}$ the corresponding foliations of $\mathcal{E}_{x}$ obtained by the identification $\mathcal{H}_{x}: W_{x} \rightarrow \mathcal{E}_{x}$. Thus we obtain the foliations of $\bar{U}^{k}$ of $\mathcal{E}$ which are invariant under the polynomial normal form maps $\mathcal{P}_{x}$. Since the maps $\mathcal{H}_{x}$ are diffeomorphisms, $\bar{U}^{k}$ are also unique fast foliations with the same contraction rates. They are characterized by

$$
\text { for } y, z \in \mathcal{E}_{x} \quad z \in \bar{U}^{k}(y) \Leftrightarrow \operatorname{dist}\left(\mathcal{P}_{x}^{n}(y), \mathcal{P}_{x}^{n}(z)\right) \leq C e^{n\left(\mu_{k}+\varepsilon\right)} \text { for all } n \in \mathbb{N}
$$

for any $\varepsilon$ sufficiently small so that $\mu_{k}+\varepsilon<\lambda_{k+1}$.

It follows from Definition 2.3 that sub-resonance polynomials $R \in \mathcal{S}_{x, y}$ are block triangular in the sense that $\mathcal{E}^{i}$ component does not depend on $\mathcal{E}^{j}$ components for $j<i$ or, equivalently, it maps the subspaces $\mathcal{V}_{x}^{i}$ of fast flag in $\mathcal{E}_{x}$ to those in $\mathcal{E}_{y}$. By considering compositions, we obtain that any sub-resonance generated polynomial in $G_{x, y}$ is also block triangular.

It is easy to see that all derivatives of a sub-resonance polynomial are sub-resonance polynomials. In particular, the derivative $D_{y} \mathcal{P}_{x}$ at any point $y \in \mathcal{E}_{x}$ is sub-resonance and hence is block triangular and thus maps subspaces parallel to $V_{x}^{k}$ to subspaces parallel to $V_{f x}^{k}$. Hence the foliation of $\mathcal{E}$ by those parallel to $V_{x}^{k}$ in $\mathcal{E}_{x}$ is invariant under the maps $\mathcal{P}_{x}$ and hence coincides with $\bar{U}^{k}$ by uniqueness of the fast foliation. It follows that for any $x \in \mathcal{M}$ and any $y \in W_{x}$ the diffeomorphism

$$
\mathcal{G}_{x, y}:=\mathcal{H}_{y} \circ \mathcal{H}_{x}^{-1}: \mathcal{E}_{x} \rightarrow \mathcal{E}_{y}
$$

maps the fast flag of linear foliations of $\mathcal{E}_{x}$ to that of $\mathcal{E}_{y}$. In particular, the same holds for its derivative $D_{0} \mathcal{G}_{x, y}=D_{x} H_{y}: \mathcal{E}_{x} \rightarrow \mathcal{E}_{y}$ and we conclude that $D_{0} \mathcal{G}_{x, y}$ is block triangular and thus is a sub-resonance linear map.

3.3. Proof of (iv): Consistency of normal form coordinates. In this section we show that the map $\mathcal{G}_{x, y}$ in (3.17) is a sub-resonance generated polynomial. First we note that

$$
\mathcal{G}_{x, y}(0)=\mathcal{H}_{y}(x)=: \bar{x} \in \mathcal{E}_{y} \quad \text { and } \quad D_{0} \mathcal{G}_{x, y}=D_{x} \mathcal{H}_{y} .
$$

Since $\mathcal{H}_{f^{n} x}^{-1} \circ \mathcal{P}_{x}^{n} \circ \mathcal{H}_{x}=f^{n}=\mathcal{H}_{f^{n} y}^{-1} \circ \mathcal{P}_{y}^{n} \circ \mathcal{H}_{y}$ we obtain that

$$
\begin{gathered}
\mathcal{H}_{f^{n} y} \circ \mathcal{H}_{f^{n} x}^{-1} \circ \mathcal{P}_{x}^{n}=\mathcal{H}_{f^{n} y} \circ f^{n} \circ \mathcal{H}_{x}^{-1}=\mathcal{P}_{y}^{n} \circ \mathcal{H}_{y} \circ \mathcal{H}_{x}^{-1} \text { and hence } \\
\mathcal{G}_{f^{n} x, f^{n} y} \circ \mathcal{P}_{x}^{n}=\mathcal{P}_{y}^{n} \circ \mathcal{G}_{x, y} .
\end{gathered}
$$


Now we consider the formal power series for $\mathcal{G}_{x, y}: \mathcal{E}_{x} \rightarrow \mathcal{E}_{y}$ at $t=0 \in \mathcal{E}_{x}$ :

$$
\mathcal{G}_{x, y}(t) \sim G_{x, y}(t)=\bar{x}+\sum_{m=1}^{\infty} G_{x, y}^{(m)}(t) .
$$

Our first goal is to show that all its terms are sub-resonance generated. We proved in Section 3.2 that the first derivative $G_{x, y}^{(1)}=D_{x} H_{y}$ is a sub-resonance linear map.

Inductively, we assume that $G_{x, y}^{(m)}$ has only sub-resonance generated terms for all $x \in \mathcal{M}, y \in W_{x}$, and $m=1, \ldots, k-1$ and show that the same holds for $G_{x, y}^{(k)}$. We split $G_{x, y}^{(k)}=S_{x, y}+N_{x, y}$ into the sub-resonance generated part and the rest. Using invariance under contracting maps $\mathcal{P}_{x}$, it suffices to show that $N_{x, y}=0$ for all $y \in W_{x}$ that are sufficiently close to $x$. Assuming the contrary, we fix such $x$ and $y$ with $N_{x, y} \neq 0$. We will write $N_{x}$ for $N_{x, y}$ and $N_{f^{n} x}$ for $N_{f^{n} x, f^{n} y}$. Now we consider order $k$ terms in the Taylor series at $0 \in \mathcal{E}_{x}$ for (3.18). Taylor series for $\mathcal{P}_{x}^{n}$ at $0 \in \mathcal{E}_{x}$ coincides with $\mathcal{P}_{x}^{n}(t)=\sum_{m=1}^{M} P_{x}^{(m)}(t)$. We also consider the Taylor series for $\mathcal{P}_{y}^{n}$ at $\mathcal{G}_{x, y}(0)=\bar{x} \in \mathcal{E}_{y}$

$$
\mathcal{P}_{y}^{n}(z)=\bar{x}_{n}+\sum_{m=1}^{M} Q_{y}^{(m)}(z-\bar{x}), \quad \text { where } \bar{x}_{n}=\mathcal{P}_{y}^{n}(\bar{x})
$$

All terms $Q^{(m)}$ are sub-resonance as the derivatives of a sub-resonance polynomial. Consider the formal power series for

$$
\mathcal{G}_{f^{n} x, f^{n} y}(t) \sim G_{f^{n} x, f^{n} y}(t)=\bar{x}_{n}+\sum_{m=1}^{\infty} G_{f^{n} x, f^{n} y}^{(m)}(t) .
$$

Now we obtain from (3.18)

$$
\bar{x}_{n}+\sum_{j=1}^{\infty} G_{f^{n} x, f^{n} y}^{(j)}\left(\sum_{m=1}^{M} P_{x}^{(m)}(t)\right)=\bar{x}_{n}+\sum_{m=1}^{M} Q_{y}^{(m)}\left(\sum_{j=1}^{\infty} G_{x, y}^{(j)}(t)\right) .
$$

Since any composition of sub-resonance generated terms is sub-resonance generated, taking non sub-resonance generated terms of order $k$ in the above equation yields

$$
N_{f^{n} x}\left(P_{x}^{(1)}(t)\right)=Q_{y}^{(1)}\left(N_{x}(t)\right) .
$$

We decompose $N$ into components $N=\left(N^{1}, \ldots, N^{l}\right)$ and let $i$ be the largest index so that $N_{x}^{i} \neq 0$, i.e. there exists $t^{\prime} \in \mathcal{E}_{x}$ so that $z^{\prime}=N^{i}\left(t^{\prime}\right)$ has non-zero component in $\mathcal{E}_{y}^{i}$. We denote

$$
w=Q_{y}^{(1)}\left(z^{\prime}\right)=D_{\bar{x}} \mathcal{P}_{y}^{n}\left(z^{\prime}\right) \in \mathcal{E}_{f^{n} y} \quad \text { and let } w_{i}=\operatorname{Pr}_{i}(w) \in \mathcal{E}_{f^{n} y}^{i}
$$

be its $i$ component. We claim that

$$
\left\|w_{i}\right\| \geq C e^{n\left(\lambda_{i}-\varepsilon\right)}
$$

where the constant $C$ does not depend on $n$. This follows from (2.3) and the fact that

$$
D_{\bar{x}} \mathcal{P}_{y}^{n}=D_{f^{n} x} H_{f^{n} y} \circ F_{x}^{n} \circ\left(D_{x} H_{y}\right)^{-1} .
$$


Indeed, the differentials $D_{f^{n} x} H_{f^{n} y}$ and $D_{x} H_{y}^{-1}$ preserve the fast flag and are close to identity since $x$ is close to $y$ by our assumption and hence $f^{n} x$ is close to $f^{n} y$. Then $z=\left(D_{x} H_{y}\right)^{-1}\left(z^{\prime}\right)$ has non-zero component $z_{i}$ in $\mathcal{E}_{x}^{i}$, which is transformed by $F_{x}^{n}$ according to (2.3) $):\left\|F_{x}^{n}\left(z_{i}\right)\right\| \geq e^{n\left(\lambda_{i}-\varepsilon\right)}\left\|z_{i}\right\|$. Then finally $\left\|w_{i}\right\| \geq C^{\prime}\left\|F_{x}^{n}\left(z_{i}\right)\right\|$ as $D_{f^{n} x} H_{f^{n} y}$ is close to identity and $F_{x}^{n}(z)$ has no $j$ components for $j>i$.

Now we estimate from above the $i$ component of the left side of (3.19) at $t^{\prime}$. First,

$$
\left\|P_{x}^{(1)}\left(t_{j}^{\prime}\right)\right\|=\left\|F_{x}^{n}\left(t_{j}^{\prime}\right)\right\| \leq\left\|\left(t^{\prime}\right)\right\| e^{n\left(\mu_{j}+\varepsilon\right)} \quad \text { for any } j .
$$

Let $N_{f^{n} x}^{s}$ be a term of homogeneity type $s=\left(s_{1}, \ldots, s_{l}\right)$ in the component $N_{f^{n} x}^{i}$. We estimate as in (3.10)

$$
\left\|N_{f^{n} x}^{s}\left(P_{x}^{(1)}\left(t^{\prime}\right)\right)\right\| \leq\left\|N_{f^{n} x}\right\| \cdot\left\|\left(t^{\prime}\right)\right\|^{k} \cdot e^{n \sum s_{j}\left(\mu_{j}+\varepsilon\right)}
$$

Since no term in $N_{f^{n} x}^{i}$ is a sub-resonance one, we have $\lambda_{i}>\sum s_{j} \mu_{j}$. We can choose a sufficiently small $\varepsilon>0$ so that $\lambda_{i}>\sum s_{j} \mu_{j}+(n+2) \varepsilon$ for all such relations. Hence the left side of (3.19) at $t^{\prime}$ can be estimated as

$$
\left\|N_{f^{n} x}^{s}\left(P_{x}^{(1)}\left(t^{\prime}\right)\right)\right\| \leq C^{\prime} e^{n\left(\lambda_{i}-2 \varepsilon\right)}
$$

where the constant $C^{\prime}$ does not depend on $n$ since the norms of $G_{f^{n} x, f^{n} y}^{(k)}$, and hence those of $N_{f^{n} x}$, are uniformly bounded. This contradicts (3.19) and (3.21) for large $n$.

Thus we shown that the Taylor series $G_{x, y}$ of $\mathcal{G}_{x, y}$ at 0 contains only sub-resonance generated terms, and in particular it is a finite polynomial. It remains to show that $\mathcal{G}_{x, y}$ coincides with its Taylor series.

In addition to (3.18) we have the same relation for their finite Taylor series

$$
G_{f^{n} x, f^{n} y} \circ \mathcal{P}_{x}^{n}=\mathcal{P}_{y}^{n} \circ G_{x, y}
$$

and hence denoting $\Delta_{n}=\mathcal{G}_{f^{n} x, f^{n} y}-G_{f^{n} x, f^{n} y}$ we obtain

$$
\Delta_{0}=\left(\mathcal{P}_{y}^{n}\right)^{-1} \circ \Delta_{n} \circ \mathcal{P}_{x}^{n}
$$

Below we show that the right hand side of (3.23) tends to 0 as $n \rightarrow \infty$ and thus $\mathcal{G}_{x, y}=G_{x, y}$, completing the proof.

Since $\Delta_{n}$ is infinitely flat at $0 \in \mathcal{E}_{f^{n} x}$, for each $k$ there exists $C_{k, \delta}$ such that

$$
\left\|\Delta_{n}(z)\right\| \leq C_{k, \delta}\|z\|^{k} \quad \text { for all } z \in \mathcal{E}_{f^{n} x} \text { with }\|z\| \leq \delta .
$$

We can choose the constant $C_{k, \delta}$ independent of $n$ since $\mathcal{G}_{x, y}$ depends continuously on $(x, y)$ in $C^{\infty}$ topology and $f^{n} x$ remains close to $f^{n} y$. We fix $k$ and $\varepsilon$ such that

$$
k \mu_{l}+2(k+1) \varepsilon<\lambda_{1}
$$

We estimate $\left(\mathcal{P}_{y}^{n}\right)^{-1}$ and $\mathcal{P}_{x}^{n}$ as follows:

$$
\left\|\left(\mathcal{P}_{y}^{n}\right)^{-1}(t)\right\| \leq e^{-n\left(\lambda_{1}-2 \varepsilon\right)}\|t\| \quad \text { and } \quad\left\|\left(\mathcal{P}_{x}^{n}\right)(t)\right\| \leq e^{n\left(\mu_{l}+2 \varepsilon\right)}\|t\|
$$


for any $n \in \mathbb{N}$ provided that sizes of both the input $\|t\|$ and the outputs $\left\|\left(\mathcal{P}_{y}^{n}\right)(t)\right\|$ and $\left\|\left(\mathcal{P}_{y}^{n}\right)^{-1}(t)\right\|$ are at most $\delta$. Both estimates follow from the fact that for any $x \in \mathcal{M}$

$$
\left\|D_{0} \mathcal{P}_{x}\right\|=\left\|F_{x}\right\| \leq e^{\mu_{l}+\varepsilon} \quad \text { and } \quad\left\|D_{0}\left(\mathcal{P}_{x}\right)^{-1}\right\|=\left\|\left(F_{x}\right)^{-1}\right\| \leq e^{-\lambda_{1}+\varepsilon}
$$

by (2.3) and hence we have similar estimates for derivatives at all points $t \in \mathcal{E}_{x}$ with $\|t\| \leq \delta$ for sufficiently small $\delta>0$ :

$$
\left\|D_{t} \mathcal{P}_{x}\right\| \leq e^{\mu_{l}+2 \varepsilon} \quad \text { and } \quad\left\|D_{t}\left(\mathcal{P}_{x}\right)^{-1}\right\| \leq e^{-\lambda_{1}+2 \varepsilon} .
$$

Then the bound for $\left\|\left(\mathcal{P}_{x}^{n}\right)(t)\right\|$ follows by simply estimating its derivative as the trajectory product, as long as all points involved are $\delta$ close to 0 .

Finally using (3.23), (3.24), and (3.26) we estimate

$$
\begin{aligned}
\left\|\Delta_{0}(t)\right\| & \leq\left\|\left(\mathcal{P}_{y}^{n}\right)^{-1}\right\| \cdot\left\|\Delta_{n} \circ \mathcal{P}_{x}^{n}(t)\right\| \leq e^{-n\left(\lambda_{1}-2 \varepsilon\right)} C_{k, \delta}\left(e^{n\left(\mu_{l}+2 \varepsilon\right)}\|t\|\right)^{k}= \\
& =e^{n\left(-\lambda_{1}+k \mu_{l}+2(k+1) \varepsilon\right)} C_{k, \delta}\|t\|^{k} \rightarrow 0 \quad \text { as } n \rightarrow \infty
\end{aligned}
$$

by (3.25). Thus, $\Delta_{0}=0$, i.e. the map $\mathcal{G}_{x, y}$ coincides with its Taylor series.

This completes the proof of Theorem 2.4

\section{REFERENCES}

[BKo] I. U. Bronstein and A. Ya. Kopanskii. Smooth invariant manifolds and normal forms. World Scientific, 1994.

[F07] Y. Fang. On the rigidity of quasiconformal Anosov flows. Ergodic Theory Dynam. Systems 27 (2007), no. 6, 1773-1802.

[FFH10] Y. Fang, P. Foulon, and B. Hasselblatt. Zygmund strong foliations in higher dimension. J. Mod. Dyn. 4 (2010), no. 3, 549-569.

[Fe95] R. Feres. The invariant connection of 1/2-pinched Anosov diffeomorphism and rigidity. Pacific J. Math. Vol. 171 No. 1 (1995), 139-155.

[Fe04] R. Feres. A differential-geometric view of normal forms of contractions. In Modern Dynamical Systems and Applications, Eds.: M. Brin, B. Hasselblatt, Y. Pesin, Cambridge University Press, (2004) 103-121.

[FiKSp11] D. Fisher, B. Kalinin, and R. Spatzier. Totally non-symplectic Anosov actions on tori and nilmanifolds. Geometry and Topology 15 (2011) 191-216.

[GoKS11] A. Gogolev, B. Kalinin, and V. Sadovskaya. Local rigidity for Anosov automorphisms. (with Appendix by R. de la Llave) Mathematical Research Letters, 18 (2011), no. 05, 843-858.

[G02] M. Guysinsky. The theory of non-stationary normal forms. Ergod. Theory Dyn. Syst., 22 (3), (2002), 845-862.

[GKt98] M. Guysinsky and A. Katok. Normal forms and invariant geometric structures for dynamical systems with invariant contracting foliations. Math. Research Letters 5 (1998), 149-163.

[HPS] M. Hirsch, C. Pugh, and M. Shub. Invariant manifolds. Springer-Verlag, New York, 1977.

[KKtR11] B. Kalinin, A. Katok, and F. Rodriguez-Hertz. Nonuniform measure rigidity. Annals of Mathematics 174 (2011), no. 1, 361-400.

[KtL91] A. Katok and J. Lewis. Local rigidity for certain groups of toral automorphisms. Israel J. Math. 75 (1991), 203-241.

[KtSp97] A. Katok and R. Spatzier. Differential rigidity of Anosov actions of higher rank abelian groups and algebraic lattice actions. Tr. Mat. Inst. Steklova 216 (1997), Din. Sist. i Smezhnye Vopr., 292319; translation in Proc. Steklov Inst. Math. 1997, no. 1 (216), 287-314. 
[KS03] B. Kalinin and V. Sadovskaya. On local and global rigidity of quasiconformal Anosov diffeomorphisms. Journal of the Institute of Mathematics of Jussieu (2003) 2 (4), 567-582.

[KS06] B. Kalinin and V. Sadovskaya. Global rigidity for totally nonsymplectic Anosov $\mathbb{Z}^{k}$ actions. Geometry and Topology, vol. 10 (2006), 929-954.

[KS07] B. Kalinin and V. Sadovskaya. On classification of resonance-free Anosov $\mathbb{Z}^{k}$ actions. Michigan Mathematical Journal, Vol 55 (2007), no. 3, 651-670.

$[\mathrm{P}]$ Ya. Pesin. Lectures on Partial Hyperbolicity and Stable Ergodicity. Zurich Lectures in Advanced Mathematics, EMS, 2004.

[PSW97] C. Pugh, M. Shub, and A. Wilkinson. Hölder foliations. Duke Mathematical Journal, 86 (1997), no. 3, 517-546.

[S05] V. Sadovskaya. On uniformly quasiconformal Anosov systems. Math. Research Letters, vol. 12 (2005), no. 3, 425-441.

[St57] S. Sternberg. Local contractions and a theorem of Poincaré. Amer. J. of Math. 79 (1957), 809-824.

Department of Mathematics, The Pennsylvania State University, University Park, PA 16802, USA.

E-mail address: kalinin@psu.edu, sadovskaya@psu.edu 